\title{
Cross Layer Preemptive Route Repair Scheme for MANET
}

\author{
Mehajabeen Fatima \\ RGPV, Bhopal, India
}

\author{
Roopam Gupta \\ RGPV, Bhopal, India
}

\author{
T.K.Bandhopadhyay \\ BIST, Bhopal, India
}

\begin{abstract}
The challenge of mobile adhoc network (MANET) is to keep the path active for long time. Since the path breaks due to node movement and limited battery power. The route is repaired and discovered only after a path breaks in existing routing protocols. Detection of route break and establishment of a new route insisted a high cost on the network. Preemptive route repair can be an alternative. It allows a routing algorithm to maintain connectivity by switching to a new path before a path break. In this paper, 'Route discovery by cross layer routing protocol ( RDCLRP)' is proposed. RDCLRP is modification of AODV. RDCLRP is a preemptive route repair routing protocol using cross layer approach. A route is considered to break when the node is moving towards out of transmission range or battery power is going to zero. When a path is about to break, a warning is broadcast showing the possibility of disconnection. A route is discovered in advance before route break to avoid disconnection. The performance of proposed algorithm is investigated for change in traffic. Simulations are run on qualnet 5.0. Proposed algorithm shows improvement compared to an AODV. Results illustrated that the route breakage is reduced by an average of $91 \%$, throughput is improved by $30.62 \%$, delay is reduced by $24.1 \%$, received packets are increased by $24.3 \%$ and residual battery power is increased by $16.05 \%$.
\end{abstract}

Keywords: MANET, AODV, route repair, route discovery, Hello Warning message (HWM).

\section{INTRODUCTION}

Mobile ad hoc network (MANET) is a infrastructureless network. It is featured by multi-hop communication, limited resources and limited security. The packets sent by the source node move through several intermediate nodes before reaching the destination node[1]. The nodes in MANET communicate through each other. The formed topology is temporary. The nature of MANET is highly dynamic. This results in random and frequent changes in the network topology. The complexity of routing among nodes is increased. Besides the feature of mobility of nodes, the specific characteristic of the wireless channel introduces more difficulties [2]. With the emerging technologies and popularity of wireless communication, number of users is increasing day by day. This also leads to more data flow and traffic in adhoc network. Nodes in an ad hoc environment face major challenge of route break due to mobility, battery power consumption and traffic. The mobility in an ad hoc network can be featured by the speed of the nodes in the systems [3]. The average speed of nodes determines the rate at which route breaks. Consequently the control traffic is used for route repairing in on-demand protocols. This increases control traffic unnecessarily. The increased routing control traffic badly affects the packet delivery ratio, power consumption, delay, throughput etc. The routing protocols must be able to adapt all changes to perform efficiently and effectively $[4,5]$. If life of a node is short then chances of link failure dominates the network. When a link failure occurs, the TCP sender reacts as if congestion was the cause. It reduces its congestion window [6]. This results in packet loss and bandwidth loss unnecessarily. This may lead to delay in communication and serious concern towards the real time applications. Real network traffic measurement and characteristics are required to analyse [7].

TCP\IP is a worldwide popular architecture, used for wired and wireless networking. TCPIIP architecture follows hierarchy and only adjacent layer can communicate with each other. The nodes are mobile and have less battery power in MANET. This dynamic nature of MANET can't be handled with TCP/IP architecture efficiently. So cross layer architecture can be used to cope with the dynamics of MANET. In cross layer design, all layers can communicate with each other. The decision can be taken on the basis of parameter of any layer resulting in quick and correct decision. [8] concluded that changing the link distance affects the network throughput. [9] Xiaoxia Huang et.al. discussed that the reactive protocols perform well when the network load is moderate. [10] analyzed that the proactive protocols perform well for heavy traffic load. The reactive routing protocols performance degrades for high traffic. It is also difficult to maintain uninterrupted communication of data with the increase in traffic, mobility, power consumption. A lot of research has been reported [11-18] addressing these issues.

One of the pioneering work on reduction of link breakage was published by T. Goff et.al. in 2001 [17]. They proposed a Preemptive routing, aims to reduce the possibility that a packet meets a broken link. In proposed algorithm, nodes monitor the link status all the time. Once a link detects bad status, the current node will send a message to the source node immediately of the route including this bad link, subsequently the source will change to proactive route. After the route hand-off, packets will be forwarded to the new and healthy route, leaving the bad link to break down silently without any packet losses. T. Goff et.al showed that the broken paths reduced considerably in comparison of DSR and AODV with the increase in preemptive ratio. In this paper only mobility is considered, other constraint resources were not considered. B R Sujatha et. al. in 2009 evaluated the DSR protocol performance under preemptive routing [18]. To discover an alternative path, preemptive message is generated. They used the signal strength threshold for preemptive route repair in their paper. It is required to consider other parameters also like speed, pause, battery power etc. Srinath 
Perur et. al. [19] in 2002, proposed a preemptive handoff strategy before link breakage wherein a node detects one of its links weakening. This prevents the route from being bro ken. But how many times route breakage is prevented is not given. [20] Wen-Hsin Yang et.al. in 2010 defined an integrated WiMAX and WiFi network architecture and discussed the corresponding handover scenarios. They proposed an energyefficient handover scheme with geographic mobility awareness (HGMA) scheme that considers the geographic mobility (GM) of mobile devices (MD). The unnecessary handovers are eliminated by reducing the number of network scanning and avoiding too frequent interface switching, the proposed HGMA scheme can significantly conserve the energy of MDs due to handover. This proposal work on only one parameter and does not consider all parameters of path disconnection. Thus, it is required to consider all parameters of route breakage to deduce the results towards real scenario

From literature survey, it is found that preemptive route repair and handoff are more promising alternate when a route is about to break. An alternative path is sought only after an active path fails. It is fine to repair the route after path fails but it increases control traffic. It is not in favor of non real time data transmission. This also hampers the continuity of data flow, causes drop of call. So it cannot be used for real time communication also. Thus, the cost of detecting a path failure is high. Therefore, it is required to design the protocol that can be used for uninterrupted real time and non real time communication both. All these reasons motivated us to investigate into this field. In this paper alternate route repair method is proposed to maintain continuous flow of traffic and to overcome the burden of traffic.

In part 2, overview of AODV is given. Part 3 discussed about proposed algorithm, Part 4 explained proposed algorithm variants. Part 5 gave results and graphs and then the conclusion is drawn in part 6 .

\section{OVERVIEW OF AD HOC ON- DEMAND DISTANCE VECTOR (AODV)}

AODV is an IETF standardizing protocol for MANET, designed by Charles E. Perkins and Elizabeth M. Royer. AODV works in two phases (i) Route Discovery (ii) Route maintenance. Route is discovered through RREQ and RREP control message. Hello message (HM), local repair, RREQ and RERR packet are used for route maintenance. [21].

Nodes which are not participating in data transmissiom neither maintain any routing information nor participate in any periodic routing table exchanges[22]. A node does not discover and maintain a route until the two need to communicate. The node provides its services as an intermediate node to maintain connectivity between two other nodes. The network connectivity of the mobile node is maintained by each mobile node when it receives RREQ packet[23]. The node can be know the other nodes in its neighborhood by the use of several broadcasts known as Hello messages (HM).

The algorithm objectives are

- To broadcast route discovery packets only when it is required.

- To distinguish between local connectivity management (neighborhood detection) and general topology maintenance.
- To disseminate information about changes to those neighbor nodes that are in requirement of information.

As soon as a node receives RREQ, that node start transmitting $\mathrm{HM}$ as shown in Fig 1. This maintains network connectivity. In this manner most of the nodes broadcast $\mathrm{HM}$ in the network. According to Ian Chakeres et. al [22], any broadcast control message serves as a HM determines network connectivity. It indicates the presence of a neighbor. If a node does not send any broadcast control message within a specified interval, a HM is broadcasted. According to Perkins and Royer, HM can also be used to detect bidirectional links $[24,25]$.

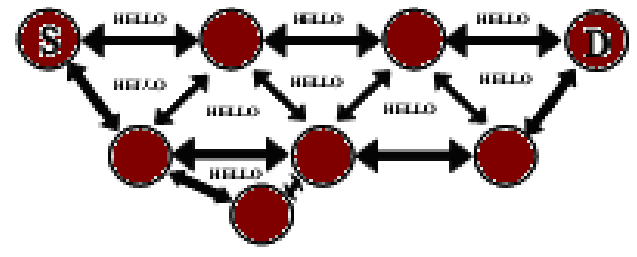

Fig.1 network connectivity through HM[14]

HM size varies from 20 bytes to 512 bytes. Ian D. Chakeres illustrates that the throughput and reception of data packets are maximum when the size of control message is equal to the size of data packets $[26,27]$. So it is recommended that keep the control packet size equal to the data packet size. AODV is simple in operation and performs well but not perfect so far. It still has many issues like Flooding for Route discovery[28], Route maintenance, Delay in finding of route [29], Power management [30], A unidirectional link problem, security like Selfish node and Malicious node etc. There are many proposals available in the literature to solve these problems. But these issues are still open, need to be amended.

\section{PROPOSED ALGORITHM}

Route maintenance is the challenge of routing protocol due to high mobility and limited battery power. Route maintenance becomes complicated because of frequent route breakage. Alternate route finding for broken link will improve the network performance. An alternative path is sought only after an active path fails. The cost of detecting a path failure is high compared to packet latencies since many retries have to time out before a path declared dead. Instead of searching path after route break A Preemptive route repair is better option for route maintenance. So

Route discovery process of proposed protocol is similar to AODV protocol i.e. route discovery is on demand, maintained by $\mathrm{HM}$ and managed by RERR control packets. In AODV protocol, if route breakage occurs near the destination, local route repair method is used. But if route breakage occurs at any other place, RERR message is send by the node to inform about the route breakage to the source node. Then the source node again start the path discovery process. In the case of large traffic, the route may break frequently incurring more flooding of RREQ control packets. This increases additional delay and overheads.

In the proposed algorithm, the route maintenance procedure of AODV during communication is modified. For this, Preemptive route repair scheme is proposed. Preemptive route repair means, a route should be repaired before route 
breakage. In AODV, RERR are generated, which move towards source node. In response source node generates Re RREQ to find new route for the same transmission. In the modified routing protocol, where route is repaired before path break, route breakage is reduced. The route is repaired locally, overhead is reduced due to local broadcast of maintenance packet in small area of network.

The convergence of the network is improved by reducing the overhead. This reduces flooding of control packets inturn reduces number of times link breakage, battery power consumption. Keeping these points in mind some modifications have been proposed in the already available AODV routing protocol.

The modifications are: route is repaired in advance before the route breakage, HM packet format is changed and it is broadcast. Surrounding nodes save active node IP address. Threshold is used for prediction of path break. When the node crosses the threshold of speed or received signal strength (RSS) or residual battery power (RBP) then that node becomes critical node and shows possibility of route break. All active nodes call speed, RSS, RBP value at the network layer and taking decision on the basis of these parameters. This is cross layer approach. New control packets named as HWM, HWR, SRM are introduced and it reduces flooding of RREQ in the network. HM are broadcast when nodes are not critical. When a node becomes critical it send HM as Hello warning message (HWM). On receiving this warning packet, if surrounding node having information about precursor and next active node will send acknowledgement as Hello warning reply (HWR). On receiving HWR, service replicate message (SRM) send to precursor, next active node and to the node who send HWR. This sets alternate hop option in the routing table for precursor or next active node and data can be transmitted via this new node without disturbing rest of the path. Using above mentioned concept three variants are possible as given in Table 1.

Table 1 Variants of proposed protocol

\begin{tabular}{|c|c|c|c|}
\hline Name & $\begin{array}{l}\text { Route } \\
\text { Discovery } \\
\text { by Cross } \\
\text { Layer } \\
\text { Routing } \\
\text { Protocol } \\
\text { with Hello }\end{array}$ & $\begin{array}{l}\text { Route } \\
\text { Discovery } \\
\text { Using Cross } \\
\text { Layer } \\
\text { Routing } \\
\text { Protocol } \\
\text { with Active } \\
\text { Nodes Hello }\end{array}$ & $\begin{array}{l}\text { Route } \\
\text { Discovery } \\
\text { Using Cross } \\
\text { Layer } \\
\text { Routing } \\
\text { Protocol } \\
\text { with Active } \\
\text { Nodes Hello } \\
\text { And Fuzzy } \\
\text { Logic }\end{array}$ \\
\hline Abbreviation & $\begin{array}{l}\text { RDCLRP- } \\
\text { HELLO }\end{array}$ & $\begin{array}{l}\text { RDCLRP- } \\
\text { ANH }\end{array}$ & $\begin{array}{l}\text { RDCLRP- } \\
\text { Fuzzy }\end{array}$ \\
\hline Procedure & $\begin{array}{l}\text { Most of the } \\
\text { nodes send } \\
\text { HM and } \\
\text { Preemptive } \\
\text { route } \\
\text { repair is } \\
\text { done if } \\
\text { speed or } \\
\text { RSS or } \\
\text { residual } \\
\text { battery } \\
\text { power } \\
\text { "(RBp) } \\
\text { crosses its } \\
\text { threshold }\end{array}$ & $\begin{array}{l}\text { Only active } \\
\text { nodes send } \\
\text { HM and } \\
\text { Preemptive } \\
\text { route } \\
\text { repair is } \\
\text { done if } \\
\text { speed or } \\
\text { RSS or } \\
\text { (RBp) } \\
\text { crosses its } \\
\text { threshold }\end{array}$ & $\begin{array}{l}\text { Only active } \\
\text { nodes send } \\
\text { HM and } \\
\text { Preemptive } \\
\text { route } \\
\text { repair is } \\
\text { done using } \\
\text { Fuzzy logic. }\end{array}$ \\
\hline
\end{tabular}

\section{PROPOSED ALGORITHM VARIANTS}

\subsection{Route Discovery by Cross Layer Routing Protocol with Hello (RDCLRP- HELLO)}

A route is discovered by RREQ and RREP. As soon as RREQ is broadcast, nodes listen it and start transmitting periodic HM. The neighbor nodes hear HM and do the entry of an IP address of neighbor nodes in its routing table. This protocol will start searching of an alternate node, if link breakage possibility is predicted in the available route. That means route repairing will be done reactively.

If Data transmission is required, broad cast RREQ in the network. The RREQ propogate in the network. When RREQ is received by a node then that node broadcast HM. Surrounding neighbor nodes hear the HM and they will store IP address of nodes in its routing table. The node which have route to destination or destination itself will send RREP. When the node receives RREP, the path is established. The node start transmitting data. Then the node check itself whether it is stationary or not. If the node is stationary then data will continue to transmit. If node is movable then node set a timer for checking of critical condition of node. When timer expires then that node will check whether the $\{$ speed of node > threshold speed or RSS > threshold RSS or Battery value $>$ threshold battery value $\}$. If condition satisfies then that node becomes critical node. The critical node broadcast HWM. The HWM is received by surrounding inactive nodes. On receiving HWM by the surrounding nodes, it checks whether IP addresses of precursor and next active node is present in its routing table or not. If IP addresses of precursor and next active node is not present in its routing table then that node will ignore the HWM. If present then, set 8th bit of Hello warning reply (HWR) message and broadcast HWR. When the critical node is received the HWR, the service replicate message (SRM) is broadcast to precursor, next node of critical node and to new node from whom HWR arrives. The SRM is received by surrounding nodes. The surrounding nodes check the SRM message whether this message is for a node or not. If yes, then replace the IP address of respective precursor or next active node IP address in its routing table with available address in SRM. If message is not for a node then Ignore HWR. On receiving SRM, replace the IP addresses of precursor or next active node, the critical node is replaced by this new node and starts sending data through new node and data can be transmitted via this node instead of critical node. In this manner route is repaired in advance before route breakage.

\subsection{Route Discovery using Cross Layer Routing Protocol with Active Nodes Hello (RDCLRP-ANH)}

$\mathrm{HM}$ is sent after every one second, it increases overhead and traffic in turn increases the possibility of congestion, consumes bandwidth unnecessary and most important consumes scarce battery power. Actually the nodes which are not participating in transmission of data, they are not required to tell about themselves. Thus it is not essential for most of the nodes to send HM. So in the proposed algorithm, only active nodes are sending HM. Because of this, power consumption of inactive nodes reduces. When these nodes 
will become active nodes later, they can utilize unused battery power and possibility of route breakage is reduced. In RDCLRP-Hello, most of the nodes send HM but in ANH, only active nodes send HM. Overhead reduces as less number of HM is broadcast and less number of link breakage reduces number of RREQ and RREP transmission. This reduces delay and increases throughput. For justification of concept results are attached. The route repair scheme is same as explained in section 4.1 except HM.

\subsection{Route Discovery using Cross Layer Routing Protocol with Active Nodes Hello and Fuzzy Logic (RDCLRP-Fuzzy)}

Path is established through RREQ and RREP. Only active nodes send HM. If speed(S) varies then a node will go out of transmission range at different times. The life of a node depends on residual battery power. The increasing or decreasing value of received signal strength (RSS) gives information about the direction of node. If the node is in critical condition then HWM is generated but this HWM is not generated instantaneously. HWM generation depends on the value of RSS, Bp and S. HWM is made adaptive. The HWM is broadcast after an interval called HWM interval (HI). This interval is decided on the basis of fuzzy logic[31]. Fuzzy logic is a tool for mapping the input features to the output based on data in the form of "IF - Then" rules controller. Here three input features are taken, namely RSS, $\mathrm{Bp}$ and S.. The key data for the system model is listed below in Tab. 3. We found that Mamdani model is applicable for our purpose, which evaluates the final output. For the application on different devices and platforms the MATLAB, fuzzy inference engine is being used. In MATLAB, ranges are added using membership function. Fuzzified input data trigger one or several rules in the fuzzy model to calculate the result. In this paper 27 rules are mapped [32]. The rule table for calculation of $\mathrm{HI}$ is given in Table 2 . The output (HI) ranges from 0 to $12 \mathrm{sec}$. in our model. We use the signal strength from 0 to $-90 \mathrm{dbm}$ (low $=-90 \mathrm{dbm}$ to $-80 \mathrm{dbm}$, medium $=-$ $80 \mathrm{dbm}$ to $-70 \mathrm{dbm}$, high $=-70 \mathrm{dbm}$ to $-60 \mathrm{dbm})$. This gives a very low potential value for all signal strength values. Simplifying, we use the speed from $0 \mathrm{mps}$ to $20 \mathrm{mps}$ and residual Battery power from $0.2 \mathrm{Ahr}$ to $0.8 \mathrm{Ahr}$ in this model. The selected ranges for $\mathrm{L}, \mathrm{M}$ and $\mathrm{H}$ are given in Table 3 . The route repair scheme is same as explained in section 4.1.

Table 2 Rule table for $\mathrm{HI}$
Table 3 Range table for $\mathrm{HI}$ calculation

\begin{tabular}{|c|c|c|c|c|}
\hline Range & $\begin{array}{c}\text { Speed(m } \\
\text { ps) }\end{array}$ & $\begin{array}{c}\text { Batter } \\
\mathbf{y} \\
\text { Power } \\
\text { (Ahr) }\end{array}$ & $\begin{array}{c}\text { Received } \\
\text { signal } \\
\text { strength(- } \\
\text { dbm) }\end{array}$ & HI(s) \\
\hline Low(L) & $0-6$ & $0.2-0.4$ & $(-60)-(-70)$ & $0-4$ \\
\hline $\begin{array}{c}\text { Mediu } \\
\text { m(M) }\end{array}$ & $6-12$ & $0.4-0.6$ & $(-50)-(-60)$ & $4-8$ \\
\hline $\begin{array}{c}\text { High(H } \\
\text { ) }\end{array}$ & $12-18$ & $0.6-0.8$ & $(-40)-(-50)$ & $8-12$ \\
\hline
\end{tabular}

\section{RESULTS AND ANALYSIS}

The impact of traffic are tested for all proposed protocol by varying the total number of sent packets from 899 packets to 4495 packets in simulation time. Simulation of the protocol is done on the qualnet simulator. Performance of new protocols is compared with basic AODV protocol. Following are the scenario parameters were constant throughout the simulation as given in table 4 . Throughput, end to end delay, packet delivery ratio, average jitter and no of times link failure during simulation has been analyzed for all the new protocols. Performance is also compared with the basic AODV protocol.

Table 4 Common Scenario Parameters

\begin{tabular}{|l|l|}
\hline \multicolumn{2}{|c|}{ Common Scenario parameters } \\
\hline No. of nodes & 100 \\
\hline Pause time & $50 \mathrm{sec}$. \\
\hline Node speed & $20 \mathrm{~m} / \mathrm{sec}$. \\
\hline Simulation time & $900 \mathrm{sec}$ \\
\hline Packet size & $512 \mathrm{bytes}$ \\
\hline Simulation Time & $900 \mathrm{sec}$ \\
\hline Channel & Path loss model \\
\hline Energy model & Micaz \\
\hline Battery model & Linear \\
\hline Mobility model & Random way point \\
\hline Start of transmission & After $1 \mathrm{sec}$. \\
\hline End of transmission & $900 \mathrm{sec}$ \\
\hline Data rate & $2 \mathrm{Mbps}$ \\
\hline Node transmission range & $399 \mathrm{~m}$ \\
\hline Simulation area & $1500 \mathrm{mX} 1500 \mathrm{~m}$ \\
\hline Number of links & 20 \\
\hline
\end{tabular}

\begin{tabular}{|c|c|c|c|c|c|c|c|c|c|}
\hline \multirow{2}{*}{$S$} & \multicolumn{2}{|c|}{$\begin{array}{c}B_{p} \\
\text { Low(L) }\end{array}$} & \multicolumn{2}{c|}{$\begin{array}{c}B_{p} \\
\text { Medium( } \\
\text { M) }\end{array}$} & \multicolumn{3}{c|}{$\begin{array}{c}B_{p} \\
\text { High(H) }\end{array}$} \\
\cline { 2 - 10 } & \multicolumn{2}{|c|}{$\boldsymbol{R S S}$} & \multicolumn{3}{c|}{$\boldsymbol{R S S}$} & \multicolumn{3}{c|}{$\boldsymbol{R S S}$} \\
\cline { 2 - 10 } & L & M & H & L & M & H & L & M & H \\
\hline L & L & L & L & H & M & H & H & H & H \\
\hline M & L & L & L & M & M & H & M & M & H \\
\hline H & L & L & L & L & L & M & L & L & M \\
\hline
\end{tabular}

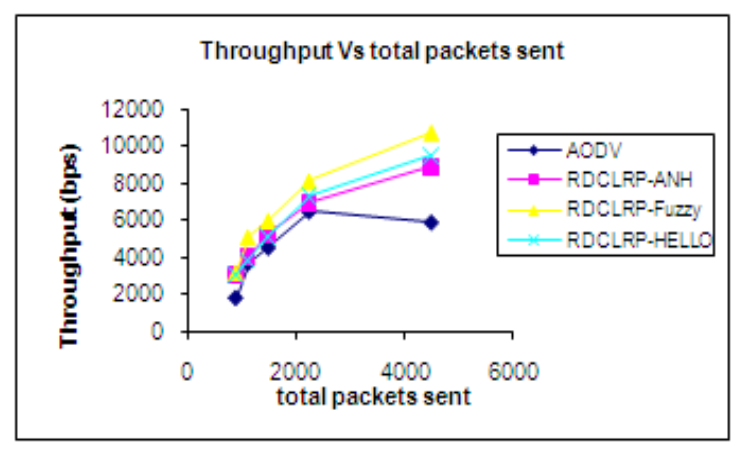

Fig.2 Throughput Vs total packets sent 


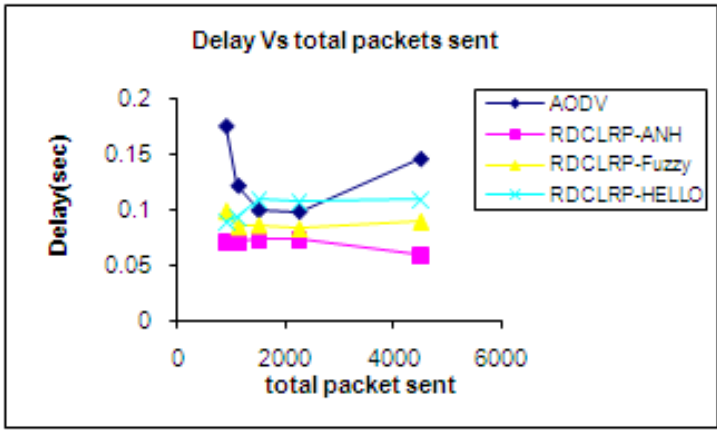

Fig.3 Delay Vs total packets sent

With the increase in total number of sent packets, traffic increases. With the increase in traffic, possibility of congestion increases. If congestion increases, packets may drop resulting in less reception of data, ultimately this decreases throughput. But if congestion is less, throughput will be more. In AODV, the congestion may occur due to increase in number of sent packets resulting in route breakage. This inturn increases control traffic resulting in less throughput. RDCLRP-HELLO, RDCLRP-ANH, RDCLRPFuzzy shows better performance compare to basic AODV as shown in Fig. 2 with the increase in traffic since the proposed variants reduces transmission of control traffic.

Proposed variants of RDCLRP shows better performance due to availability of alternate route before link breakage in comparison of AODV. The throughput is more in all variants of RDCLRP as compared to basic AODV. The overall throughput is highest in RDCLRP-Fuzzy as shown in Fig. 2, it is because of transmission of less number of control packets, replacement of critical node with available neighbor node and switching of route before route breakage. Similarly the number of received packets is highest in RDCLRP- Fuzzy as shown in Fig.4.

In the case of AODV protocol, if route breaks near the destination, local route repair method is used. But if route error occurs at any other place in between the route, RERR message is generated by the node detecting the link break to the source node. Then the source node reinitiates the path discovery process. This increases control traffic in the network. If route breaks frequently, the situation will be exploding. So the packets would take more time to reach to destination results in more delay as shown in Fig.3. RDCLRP reduces possibility of route breakage and also reduces propagation of control packets. That's why, RDCLRP variants shows better performance than the AODV. RDCLRP Fuzzy is best among all variants.

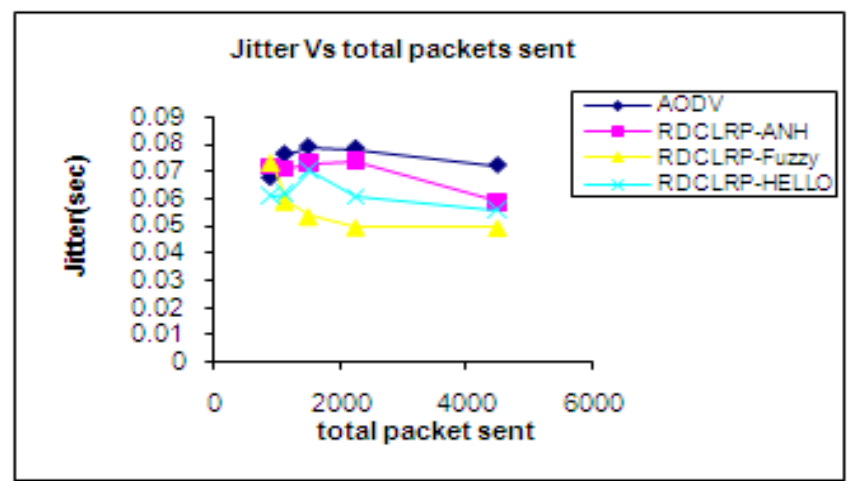

Fig.4 Jitter Vs total packets sent

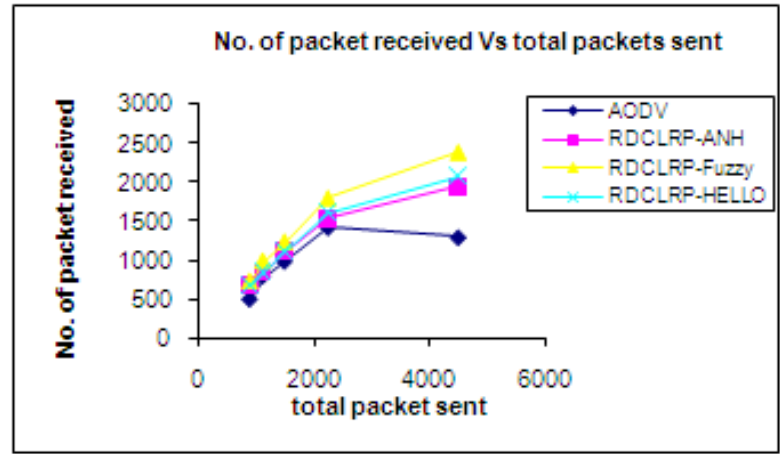

Fig.5 No. of packets received Vs total packets sent

Jitter is the time taken by two packets reached to a node, ,caused by network congestion, queue, route switching. It should be less for a routing protocol to perform better. Congestion increases with the decrease in packet interval and packet may go into the queue. This causes delay and jitter. The average jitter is shown in figure 4 . The RDCLRP variants are performed better with increase in traffic than AODV as route change is less in proposed variants.

In case of higher traffic, no. of received packets are more. The no. of received packets is more in proposed algorithms as compared to basic AODV. In case of AODV, some nodes run out of battery or go out of transmission range during the simulation and hence they could not forward packets any more. But in proposed algorithms, the bad status route is replaced by healthy route before route break, so the nodes are able to forward packets for a long period of time. Thus the number of received packets are more for proposed algorithms in comparison of AODV as shown in Fig 5. AODV also has highest no. of link breakage as shown in Fig. 7.

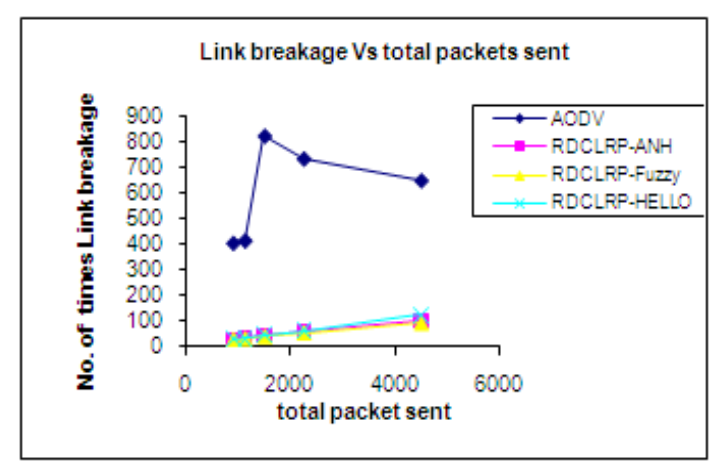

Fig.7 No. of times link breakage Vs total packets sent

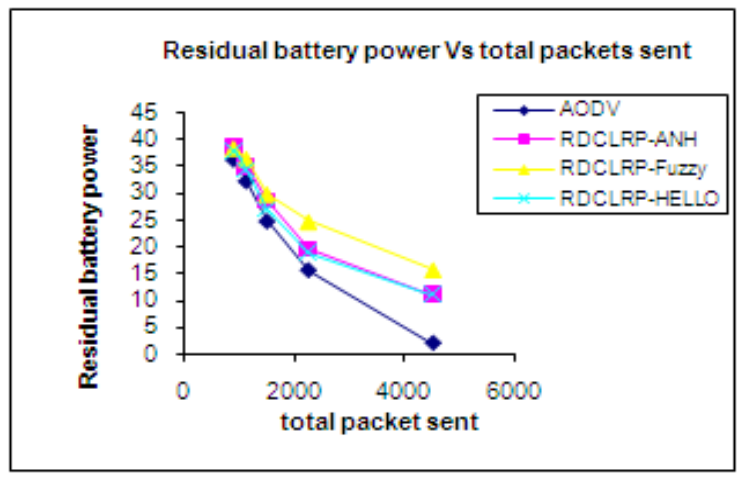

Fig.8 Residual battery power Vs total packets sent 
Links may break during the transmission of data due of movement of intermediate nodes or may be because of power failure of intermediate nodes. The AODV algorithm intimate the source about the route breakage but it does not try to reduce the possibility of route breakage. Therefore, route breakage is more in AODV compared to RDCLRP-HELLO, RDCLRP-ANH, RDCLRP-Fuzzy as shown in Fig. 7. More traffic results in frequent link breakage inturn results in the transmission of more control packets for route repair in AODV that's why the number of times link breakage and residual battery power is least in AODV as shown in Fig. 7 and 8. All proposed variants of RDCLRP have improved in all performance parameters over AODV. Number of times link breakage is minimized, so residual battery power is increased, in turn delay and jitter is minimum which increases throughput. Improvement is maximum in RDCLRP- Fuzzy among all proposed protocols. So RDCLRP- Fuzzy is best among all.

\section{CONCLUSION}

We introduce improved algorithms based on preemptive route repair are called as RDCLRP-HELLO, RDCLRP-ANH and RDCLRP-Fuzzy. According to RDCLRP, critical node can be replaced by a neighbor node before it goes out of coverage or before it dies. The performance of these algorithms are investigated for change in traffic and compared with basic AODV. It is seen that the preemptive route repair method improves the performance of network for increase in traffic. Simulation results deduce that throughput, end to end delay, route breakage, residual battery power improves for proposed algorithm in comparison of AODV. Results show that the number of times link breakage is least in RDCLRP- Fuzzy and maximum in AODV. RDCLRP- ANH has improvement of $16.64 \%$, RDCLRP- Fuzzy has improvement of $30.64 \%$ and RDCLRP- HELLO has $21.06 \%$ improvement as compared to AODV for throughput. All parameters are improved for proposed variants but highest improvement is shown by RDCLRP-Fuzzy. The proposed algorithm can be used for real time communication.

\section{REFERENCES}

[1] Marco Fotino and Floriano De Rango, "Energy Issues and Energy aware Routing in Wireless Ad-hoc Networks", International Journal of Communication System, Mobile Ad-Hoc Networks: Protocol Design, pp. 281-296, 2010

[2] Anelise Munaretto, Mauro Fonseca, "Routing and quality of service support for mobile ad hoc networks" Computer Networks, Elsevier, 2007, 3142-3156.

[3] D.B. Johnson, D.A. Maltz, "Dynamic Source Routing in AdHoc Wireless Networks", Kluwer Academic, 1996.

[4] The performance impact of traffic patterns on routing protocols in mobile ad hoc networks, Himabindu Pucha, Saumitra M. Das, Y. Charlie Hu, journal of computer networks, Elsevier, 2007.

[5] Wenjing YANG, Xinyu YANG, Guozheng LIU, Chiyong DONG, "A Bandwidth Aware Multi-path Routing Protocol in Mobile Ad Hoc Networks", Journal of Computational Information Systems, 685-696, 2011.

[6] Zhen-Jiang Zhang,1 Jun-Song Fu,1 and Han-Chieh Chao, "An Energy-Efficient Motion Strategy for Mobile Sensors in Mixed Wireless Sensor Networks"
International Journal of Distributed Sensor Networks Volume 2013.

[7] Xiao-hu G, Yang Yang, Cheng-Xiang Wang, YingZhuang Liu, Chuang Liu and Lin Xiang, "Characteristics analysis and modeling of frame traffic in 802.11 wireless networks", WIRELESS COMMUNICATIONS AND MOBILE COMPUTING, 2010, pp: 584-592.

[8] Xiaoxia Huang, Yuguang Fang, "Performance Study of Node-Disjoint Multi-path Routing in Vehicular MANETs Networks", IEEE Transactions on Vehicular Technology,vol. 58, pages 1942-1950, May 2009.

[9] Xiaoxin Wu, Gang Ding, and Wenwu Zhu, "Load-Based Route Discovery Through Searching Range Adaptation for MANET throughput Improvement", IEEE Transactions on Vehicular Technology, VOL. 58, NO. 4, MAY 2009.

[10] S. Iyer, S. Bhattacharyya, N. Taft, N. McKeoen, and C. Diot. A measurement based study of load balancing in an IP backbone. SprintATL, Tech.Rep.TR02-ATL-051027, May 2002.

[11] K. G. Larsen, P. Pettersson, and W. Yi. Uppaalin a Nutshell, International Journal on Software Tools for Technology Transfer, 1(1-2):134-152, 1997

[12] Sibusisiwe Chiyangwa, Marta Z. Kwiatkowska. A Timing Analysis of AODV, Formal Methods for Open Object-Based Distributed Systems, 7th IFIP WG 6.1 International Conference, FMOODS, Athens, Greece, June 15-17, 2005.

[13] Aleksandr Huhtonenoccurs, "Comparing AODV and OLSR Routing Protocols, , Seminar on Internetworking, Sjökulla, 2004-04-26/27.

[14] Bong Jun Choi and Xuemin (Sherman) Shen Adaptive Exponential Beacon Period Protocol for Power Saving in Delay Tolerant Networks, IEEE Communications Society, IEEE ICC 2009 proceedings

[15] Lan Guoqing, Qu Zhaowei, "Unidirectional link problem in AODV routing protocol", Broadband Network \& Multimedia Technology, IC-BNMT '09. 2nd IEEE International Conference on, 18-20 Oct., 2009.

[16] Zhu Ji, Wei Yu, And K.J. Ray Liu, “A Belief Evaluation Framework In Autonomous MANETS Under Noisy And Imperfect Observation: Vulnerability Analysis And Cooperation Enforcement", IEEE transactions on mobile computing, VOL. 9, NO. 9, September, 2010

[17] T. Goff, N. B. Abu-Ghazaleh, and D. S. Phatak, "Preemptive routing in ad hoc networks", Proc. of ACM SIGMOBILE, 2001, pp.43-52.

[18] B R Sujatha, M V Satyanarayana, -Improved Network Connectivity in MANETs, International Journal of Computer Networks \& Communications (IJCNC), Vol.1, no.3, October 2009, pp. 1-8.

[19] Srinath Perur, Abhilash P. and Sridhar Iyer, -Router Handoff: A Preemptive Route Repair Strategy for AODV,$\|$ proc. of the IEEE International Conference on Personal Wireless Communications, New Delhi, India, 15-17 December 2002, pp.168-171. 
[20] Wen-Hsin Yang, You Chiun Wang, Yu-Chee Tseng and Bao-Shuh P. Lin., "Energy-Efficient Network Selection with Mobility Pattern Awareness in an Integrated WiMAX and WiFi Network", s.l. : International journal of communication system, Volume 23, Number 2, February 2010, pp. 213-230.

[21] Perkins, C.E. et al., "Performance comparison of two ondemand routing protocols for ad hoc networks", IEEE Personal Communications, 2001.

[22] Ian D. Chakeres, Elizabeth M. Belding-Royer, AODV Routing Protocol Implementation Design, , International Conference on Distributed Computing Systems ICDCS(Workshop), pp. 698-703, 2004.

[23] C. Perkins, E. Belding-Royer and S. Das, "Ad hoc OnDemand Distance Vector (AODV) Routing", Request for Comments: 3561, July 2003.

[24] Ian D. Chakeres, Elizabeth M. Belding-Royer, "The Utility of HMs for determining Link Connectivity", International Symposium on Wireless Personal Multimedia Communications - WPMC , vol. 2, pp. 504 508, 2002.

[25] Charles E Perkins and Elizabeth M Royer, Adhoc On Demand Distance Vector Routing, proceedings of the 2nd IEEE workshop on mobile computing systems and applications, 1997.

[26] Elizabeth M. Royer and Charles E. Perkins. "An Implementation Study of the AODV Routing Protocol." Proceedings of the IEEE Wireless Communications and Networking Conference, Chicago, IL, September 2000.
[27] Preetha K G, Unnikrishnan and K Poulose Jacob, “A probabilistic approach to reduce the route establishment overhead in aodv algorithm for MANET", International journal of distributed and parallel systems (ijdps) vol.3, no.2, march 2012.

[28] K. G. Larsen, P. Pettersson, and W. Yi. Uppaalin a Nutshell, International Journal on Software Tools for Technology Transfer, pp. 134-152, 1997

[29] Sibusisiwe Chiyangwa, Marta Z. Kwiatkowska. A Timing Analysis of AODV, Formal Methods for Open Object-Based Distributed Systems, 7th IFIP WG 6.1 International Conference, FMOODS, Athens, Greece, June 15-17, 2005.

[30] Zhu Ji, Wei Yu, And K.J. Ray Liu, “A Belief Evaluation Framework In Autonomous MANETS Under Noisy And Imperfect Observation: Vulnerability Analysis And Cooperation Enforcement", IEEE transactions on mobile computing, VOL. 9, NO. 9, September, 2010

[31] RDCLRP-Route Discovery by Cross Layer Routing Protocol for Performance Improvement. Mehajabeen Fatima, Roopam Gupta, T.K. Bandhopadhyay. Kanyakumari, India. s.1. : International Conference on Network and Computer Science, April 2011, Vols. V5306

[32] Route Discovery by Cross Layer Approach for MANET - Mehajabeen Fatima, Roopam Gupta, and T.K. Bandhopadhyay. s.1. : International Journal of Computer Applications, Vols. Volume 37- No.7, pp. 0975 - 8887, January 2012. 